\title{
DIALOG AND DISCUSSION
}

\section{Editorial}

\section{KELLY A JOYNER, JR}

The recently published article 'Heparin-Induced Thrombocytopenia (HIT): A Case Study' contains several issues that I feel need to be addressed. This article does a fine job of describing a very fulminant case of HIT where the patient died following a series of missed diagnoses as well as some questionable therapy practices. This article states in several places that the use of low molecular weight heparins (LMWH) has an effective use in anticoagulation therapy, but also states that these LMWH therapies do not need to be monitored by laboratory methods. Unfractionated heparin (UF) is commonly used in anticoagulant therapy and is monitored by a variety of methods including activated partial thromboplastin time (aPTT), anti-Xa assays, and during surgery or other invasive procedures by the activated clotting time (ACT). The initiation of LMWH therapy is typically used in patients already in higher risk morbidity categories such as those with heparin sensitivity issues, those with described clotting diatheses, in higher risk pregnancies (antiphospholipid antibody patients, pre-eclamptic), and patients with histories of hypercoagulable states. It makes logical sense that drugs with similar uses and effects (UF) heparins should be monitored for better patient outcome. Patients with prolonged baseline aPTT assays, those with complex anticoagulation diagnoses, those with multiple hypercoagulable states, and high risk patients receiving anticoagulant therapy with UF and/or LMWH should be monitored using an applicable and documented assay or assays.

The ability to monitor/evaluate possible HIT status is a definite plus for any hospital, but many are limited by resources and technological capabilities. Serotonin release assay (SRA), although a sensitive and specific test, is performed by only a very few laboratories and turn around time for the assay may make this result a moot point. The platelet factor 4 assays, ELISA based, are also very sensitive and somewhat specific assays, but again technological and expense restraints may make these not profitable or standardized (i.e., heparin reversal of positive results, cost per run if only one or two patients are tested, finding a proper proficiency testing cohort).

The use of direct thrombin inhibitors (DTI) such as Lepirudin or Argatroban is becoming more widely accepted as means of anticoagulating HIT and other hypercoagulable patients. There are laboratory and medical restraints with this class of drugs such as there is typically no good or standardized method of testing anticoagulation status, there is no antidote (such as protamine sulfate for the heparins) and renal or liver dysfunction may make these drugs not viable.

The author should be commended for preparing a case study for a difficult anticoagulation scenario, but must be careful in suggesting treatment and laboratory testing methods that are not necessarily proven.

Respectfully,

Kelly A. Joyner, Jr. MS, MT(ASCP)SH, PA-C

Analytical Specialist, Clinical Coagulation Laboratory (Retired)

Duke University Medical Center

Independent Haemostasis Consultant

Rougemont, NC 